\title{
BIONANOCOMPÓSITOS HÍBRIDOS FORMADOS POR HIDROGÉIS DE POLISSACARÍDEO E NANOARGILA: I. CARACTERIZAÇÃO FÍSICO-QUÍMICA
}

\author{
Uilian Gabaldi Yonezawa ${ }^{1}$, Marcia Regina de Moura ${ }^{2}$, Fauze Ahmad Aouada ${ }^{2 *}$
}

\footnotetext{
${ }^{1}$ Doutorando no Programa de Pós-Graduação em Ciência dos Materiais, Universidade Estadual Paulista (UNESP), Faculdade de Engenharia, Câmpus de Ilha Solteira - SP.

${ }^{2}$ Professor(a) do Departamento de Física e Química, Universidade Estadual Paulista (UNESP), Faculdade de Engenharia, Câmpus de Ilha Solteira - SP. *E-mail: faouada@yahoo.com.br
}

RESUMO: O objetivo deste trabalho foi sintetizar bionanocompósitos híbridos a partir da acrilamida, polissacarídeo carboximetilcelulose e nanoargila e avaliar as propriedades físicoquímicas, tais como: hidrofílicas, cinéticas, morfológicas e espectroscópicas. Os hidrogéis foram preparados fixando as concentrações de acrilamida em $6 \% \mathrm{~m} / \mathrm{v}$ e carboximetilcelulose em $1 \% \mathrm{~m} / \mathrm{v}$, e incorporando diferentes concentrações de nanoargila (cloisita- $\left.\mathrm{Na}^{+}\right)(0-20 \%$ $\mathrm{m} / \mathrm{v}$ ). A técnica de difração de raios-x permitiu notar que as plaquetas de nanoargila se encontram intercalada na matriz polimérica. A técnica de microscopia eletrônica de varredura indicou uma morfologia de estruturas porosas, com formas bem definidas e tamanhos de poros heterogêneos. A análise térmica evidenciou uma estabilidade térmica abaixo de $200{ }^{\circ} \mathrm{C}$ para todas as amostras analisadas. Os resultados das propriedades hidrofílicas e cinéticas em água destilada e em soluções salinas indicaram que as concentrações de nanoargila e o efeito da carga iônica influenciam o intumescimento e os parâmetros cinéticos. A constante de perda de água indicou que a presença de nanoargila proporcionou uma liberação controlada de água destilada. Portanto, as propriedades físico-químicas indicam que estes bionanocompósitos híbridos são uma alternativa promissora para aplicação na agricultura, sendo sua potencialidade investigada na parte II deste trabalho.

Palavras-chave: Hidrogéis. Nanoargila. Agricultura.

\section{HYBRID BIONANOCOMPOSITES FORMED FROM POLYSACCHARIDE HYDROGELS AND NANOCLAY: I. PHYSICOCHEMICAL CHARACTERIZATION}

\begin{abstract}
The objective of this study was to synthesize hybrid bionanocomposites formed from acrylamide, carboxymethylcellulose polysaccharide and nanoclay and evaluate their physicochemical properties, such as: hydrophilic, kinetic, morphological and spectroscopic. The hydrogels were prepared by fixing of the concentrations of acrylamide in $6.0 \mathrm{wt}-\%$ and carboxymethylcellulose $1.0 \mathrm{wt}-\%$, and incorporating different nanoclay concentrations (cloisita-Na $\left.{ }^{+}\right)(0-20 \mathrm{wt}-\%)$. X-ray diffraction technique allows to observe that the nanoclay platelets are intercalated into polymeric matrix. Scanning electron microscopy technique showed porous structure morphologies with well-defined shapes and heterogeneous pore dimensions. Thermal analysis indicated a good thermal stability below $200{ }^{\circ} \mathrm{C}$ for all
\end{abstract}

Cultura Agronômica, Ilha Solteira, v.26, n.2, p.142-157, 2017 
samples. The results of hydrophilic and kinetic properties in distilled water and saline solutions showed that the concentration of nanoclay and the effect of ionic charge influenced these properties. In addition, the water loss constant indicated that the presence of nanoclay provided the water desorption of more controlled way. Therefore, the physicochemical properties indicate that these hybrid bionanocomposites are a promising alternative for applications in agriculture, being their potentiality investigated in the part II of this work.

Key words: Hydrogels. Nanoclay. Agriculture.

\section{INTRODUÇÃO}

Dentre as diversas pesquisas voltadas para o ramo agronômico, o uso do hidrogel biodegradável para fins agrícolas é uma vertente crescente e extremamente promissora e ainda muito pouco explorada. Neste contexto o emprego do hidrogel como condicionador de solo objetiva aumentar a capacidade de retenção de água e pode atuar como regulador na liberação de insumos agrícolas, minimizando assim, custos e contaminação ambiental (MARQUES et al., 2013; MAZIAD et al., 2016).

Os hidrogéis são polímeros hidrorretentores, constituídos por redes tridimensionais reticuladas e insolúveis, que são capazes de reter e absorver grandes quantidades de água (SAVI et al., 2014). O processo de intumescimento dos hidrogéis depende da presença de grupos hidrofílicos, $\mathrm{pH}$, intensidade iônica, temperatura e composição de solvente. (BARDAJEE et al., 2014; CHAUHAN, 2015).

A combinação de polímeros naturais na matriz polimérica tem como intuito obter hidrogéis com maior hidroficilidade, biodegrabilidade e biocompatibilidade. No entanto a inserção de argilominerais também tem despertado grande interesse, por melhorar a capacidade de troca catiônica, estabilidade térmica, capacidade de absorção e velocidade de absorção de água. Ao longo dos últimos anos, bionanocompósitos a base de argilominerais e polissacarídeos vêm sendo estudados para diversas aplicações, atraindo grande atenção para a agricultura e horticultura, devido a sua alta retenção de água, assim como, a sorção e liberação controlada de agrotóxicos (AHMED, 2015; LI et al., 2009; VUNDAVALLIA et al., 2015; RASHIDZADEH; OLAD, 2014; MALESU et al., 2011).

Nanocompósito sintetizado com poliacrilamida, poli(ácido acrílico) e nanoargila para aplicação na agricultura a fim de desenvolver um produto sustentável, proporcionou excelente capacidade de retenção de água demonstrando ser um produto promissor para fins agrícolas (VUNDAVALLI et al., 2015). Segundo Rashidzadeh e Olad (2014) sintetizaram nanocompósitos a base de alginato de sódio, ácido acrílico, poliacrilamida e montmorilonita via polimerização radical livre. Os autores concluíram que o nanocompósito sintetizado tem excelente perfil de liberação lenta e uma boa capacidade de retenção de água.

Nesse sentido, os principais objetivos deste trabalho foram sintetizar hidrogéis nanoestrututrados biodegradáveis baseados na carboximetilcelulose (CMC), nanoargila 
(cloisita- $\mathrm{Na}^{+}$) e acrilamida para uma possível aplicação na agricultura. E analisar as propriedades hidrofílicas, cinéticas, morfológicas e espectroscópicas. Vale ressaltar que tais hidrogéis nanoestruturados foram aplicados e analisados em um determinado cultivo (resultados apresentados na parte II).

\section{MATERIAL E MÉTODOS}

\section{Síntese do hidrogel e dos bionanocompósitos nanoestruturados}

Os experimentos foram realizados na Universidade Estadual Paulista "Júlio de Mesquita Filho", localizado em Ilha Solteira, estado de São Paulo.

Hidrogéis de poliacrilamida (PAAm), CMC (Synth) e nanoargila (Southern Clay Products) foram obtidos por meio de polimerização via radical livre. Os hidrogéis foram preparados a partir de acrilamida (Vetec) $6 \% \mathrm{~m} / \mathrm{v}, \mathrm{CMC} 1 \% \mathrm{~m} / \mathrm{v}$ e diferentes concentrações de nanoargila: 0, 10 e $20 \%$. Para a obtenção do hidrogel foram adicionados em solução aquosa de CMC, acrilamida e nanoargila, $2 \%$ de metilenobisacrilamida (MBAAm) (Vetec) como agente de reticulação e N, N, N', N' - tetrametilenodiamida (TEMED) (Sigma-Aldrich) $(6,67 \mathrm{mmol} / \mathrm{L})$ como catalisador. Em seguida, gás nitrogênio $\left(\mathrm{N}_{2}\right)$ foi borbulhado na solução por 20 minutos, e por fim foi adicionado persulfato de sódio (Sigma-Aldrich) (3,50 mmol/L) como iniciador (AOUADA, et al., 2009). Após a polimerização, os hidrogéis foram purificados com água destilada durante 5 dias, sendo que a água foi trocada a cada 24 horas. Nesse processo, reagentes que não foram polimerizados ou que não participaram da reação são eliminados.

\section{Difração de raios-x (DRX)}

Os espectros de difração de raios-x foram obtidos num difatômetro Shimadzu (modelo XRD-6000) com fonte de raios-X de $\mathrm{Cu}$; intervalo de 4 a $50^{\circ}$ e velocidade de varredura de $1 \%$ min. Utilizaram-se amostras de nanoargila, hidrogel puro e bionanocompósitos contendo 10 e $20 \%$ de nanoargila pulverizadas. Vale ressaltar que o espaçamento interplanar (d) das amostras foi determinado pela lei de Bragg.

\section{Microscopia eletrônica de varredura (MEV)}

As propriedades morfológicas foram investigadas utilizando a técnica de microscopia eletrônica de varredura através do microscópio ZEISS (modelo EVO/LS15). Amostras do hidrogel de poliacrilamida com CMC e dos hidrogéis nanoestruturados com 10 e $20 \%$ de nanoargila foram intumescidas em água destila até o equilíbrio e congelados em nitrogênio líquido. Em seguida, as amostras foram liofilizadas através do liofilizador Terroni ${ }^{\circledR}$ (modelo LT1000) durante 24 horas, com temperatura de aproximadamente $-55{ }^{\circ} \mathrm{C}$ e por fim, as amostras foram depositadas em um porta amostra e recobertas com uma fina camada de ouro.

Cultura Agronômica, Ilha Solteira, v.26, n.2, p.142-157, 2017 


\section{Análise térmica}

As curvas termogravimétricas e termogravimétricas diferenciais foram obtidas por meio do equipamento TA Instruments TGA modelo Q-500. Foram utilizadas aproximadamente 8 mg de amostra, sendo essas depositados em um porta amostra de platina, e em seguida aquecidas até $800{ }^{\circ} \mathrm{C}$ com uma taxa de aquecimento de $10{ }^{\circ} \mathrm{C}$ min. ${ }^{-1}$, com vazões de gás nitrogênio na balança de $40 \mathrm{~mL} / \mathrm{min}$ e na amostra de $60 \mathrm{~mL} / \mathrm{min}$.

\section{Grau de intumescimento (Q)}

A capacidade de absorção dos hidrogéis (0,10 e $20 \%$ de nanoargila) foi investigada a partir de medidas de grau de intumescimento em água destilada e em soluções salinas com concentrações e cargas iônicas diferentes. Foram preparadas soluções de $\mathrm{NaCl}(0,025 ; 0,05$; $0,10 ; 0,15$ e $0,20 \mathrm{~mol} / \mathrm{L}), \mathrm{CaCl}_{2}$ e $\mathrm{AlCl}_{3}(0,15 \mathrm{~mol} / \mathrm{L})$. Dada a equação, o grau de intumescimento $(\mathrm{Q})$ foi calculado através da massa do hidrogel intumescido $\left(\mathrm{M}_{\mathrm{t}}\right)$ pela massa do hidrogel seco $\left(\mathrm{M}_{\mathrm{s}}\right)$.

Para determinação de $\mathrm{Q}$, os hidrogéis foram cortados em forma cilíndrica e secos em estufa com temperatura controlada de $30^{\circ} \mathrm{C}$. Após essa etapa, os mesmos foram pesados em uma balança analítica e posteriormente colocados para intumescer. Para cada medida, as amostras foram retiradas do meio de intumescimento e pesado para obtenção da massa em tempos pré-determinados.

\section{Parâmetros cinéticos}

Os parâmetros cinéticos consistiram no processo de intumescimento envolvendo o mecanismo de absorção de água destilada e em soluções salinas com diferentes cargas iônicas. Tais parâmetros foram calculados por meio da equação (RITGER; PEPPAS, 1987).

$$
\frac{\mathrm{M}_{\mathrm{t}}}{\mathrm{M}_{\mathrm{eq}}}=\mathrm{k} \cdot \mathrm{t}^{\mathrm{n}}
$$

Onde $\mathrm{M}_{\mathrm{eq}}$ é massa do hidrogel intumescido no equilíbrio, $\mathrm{t}$ é o tempo, $\mathrm{k}$ é conhecida como constante de difusão e n é o expoente difusional, que fornecem informações sobre o tipo de mecanismo de difusão.

O estudo de difusão da água e soluções salinas para o interior da matriz dos hidrogéis foi realizado através das análises dos valores do coeficiente de difusão $(\boldsymbol{D})$, obtidos por meio da equação abaixo:

$$
\mathrm{D}=\pi \cdot \mathrm{r}^{2}\left(\frac{\mathrm{k}}{4}\right)^{\frac{1}{\mathrm{n}}}
$$

Onde D é dado $\mathrm{em}^{2} / \mathrm{h}$ e r é o raio do hidrogel seco $(\mathrm{cm})$. Para cada ensaio (realizado em triplicata), o valor de $\mathrm{r}$ foi medido antes de se iniciarem os experimentos (AOUADA, et al., 2009).

Cultura Agronômica, Ilha Solteira, v.26, n.2, p.142-157, 2017 


\section{Perda de água}

O hidrogel foi intumescido em água destilada até o estado de equilíbrio. Em seguida cortado em forma cilíndrica e seco em estufa em temperatura controlada de $30{ }^{\circ} \mathrm{C}$. Durante as primeiras 9 horas, a variação da massa das amostras foi analisada a cada 1 hora. Após este período, a massa foi pesada em períodos de tempos maiores. Os resultados das análises de perda de água (\%) foram obtidos através da razão entre a variação da massa, $\mathbf{M}_{\mathrm{eq}} \mathbf{M}_{\mathrm{t}}$, pela massa intumescida em equilíbrio, $\mathrm{M}_{\mathrm{eq}}$.

\section{RESULTADOS E DISCUSSÃO}

A técnica de difração de raios-x foi utilizada para avaliar a conformação das plaquetas de nanoargila nos bionanocompósitos. Ao avaliar o espaçamento basal da nanoargila pura e dos bionanocompósitos é possível analisar a disposição das plaquetas de nanoargila na matriz do polímero (intercalada ou esfoliada) utilizando a lei de Bragg. As Figuras 1a e 1b mostram os difratogramas de raios-x da nanoargila pura, hidrogel puro e dos bionanocompósitos contendo 10 e $20 \%$ de nanoargila.

O difratograma correspondente a nanoargila pura demonstra um espaçamento basal de 1,19 nm (Figura 1b), próximo ao espaçamento basal teórico de 1,17 nm (MALLAKPOUR; DINARI, 2012). Pode-se observar ainda picos em $2 \theta$ em 19, $7^{\circ}, 21,9^{\circ}$ e 28, $2^{\circ}$ correspondentes a esmectita (E) e quartzo (Q) (GONÇALVES et al., 2014). Com relação ao difratograma do hidrogel puro (PAAm e CMC) não se observou nenhum pico cristalino, evidenciando ser uma estrutura amorfa (Figura 1a), como esperado.

Pode-se observar nos difratogramas das amostras dos bionanocompósitos um deslocamento do pico de difração para ângulo mais baixo $\left(2 \theta=6,16^{\circ}\right)$ com relação à nanoargila pura $\left(2 \theta=7,40^{\circ}\right)$. Além disso, nota-se que o espaçamento basal dos bionanocompósitos aumentou para $1,44 \mathrm{~nm}$ quando comparado com a nanoargila pura 1,19 $\mathrm{nm}$, o que indica que a nanoargila se encontra intercalada na matriz do hidrogel (Figura 1b).

A investigação morfológica do hidrogel sintetizado com poliacrilamida e CMC e dos bionanocompósitos constituídos de poliacrilamida, CMC e nanoargila (10 e $20 \%$ ) foram realizadas por medidas de microscopia eletrônica de varredura. 

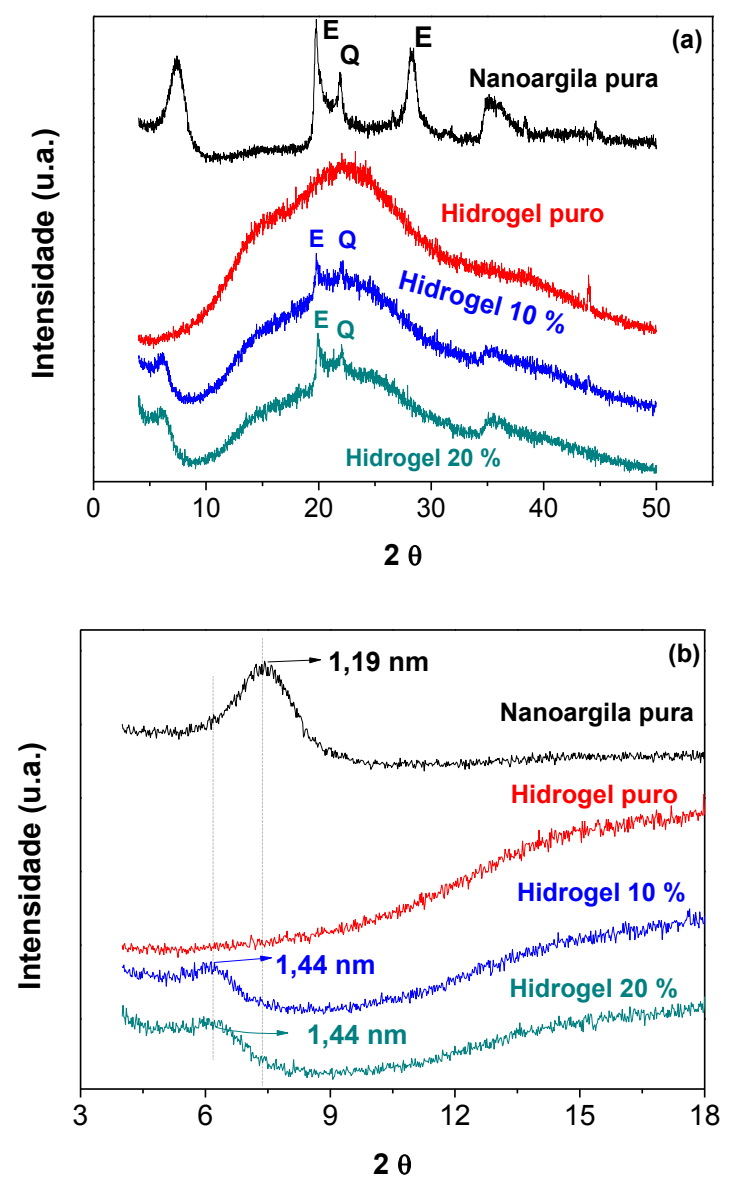

Figura 1. (a) Difratogramas de raios- $x$ da nanoargila pura, hidrogel puro e dos bionanocompósitos contendo diferentes concentrações de nanoargila e (b) Difratogramas expandidos na região de $2 \theta=4-18^{\circ}$ mostrando a intercalação das camadas de nanoargila.

Observou-se que todas as amostras possuem estruturas básicas semelhantes. Nota-se também uma diminuição nos tamanhos dos poros com o incremento da nanoargila no material (Figura 2) o que evidencia que a nanoargila pode estar atuando como possível reticulador físico e/ou químico (NASCIMENTO et al., 2017). Vale ressaltar que hidrogéis com poros grandes tem forte interação com a água, proporcionando maior absorção de água. Por outro lado, hidrogéis com poros pequenos terão uma menor área de superfície média por poro em contato com a água, o que acarretará em uma baixa hidrofilicidade (SAPUTRA et al., 2015). 

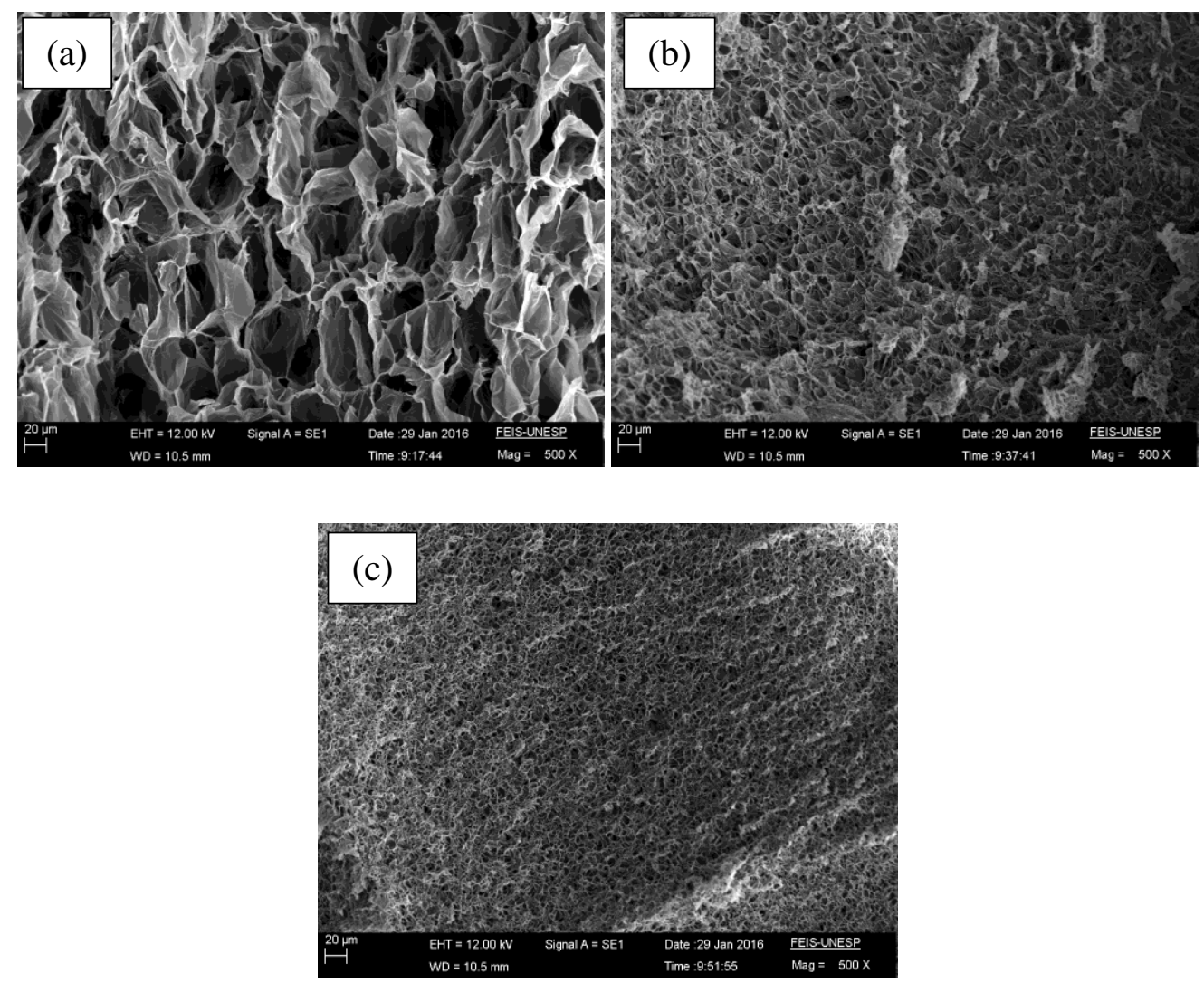

Figura 2. Micrografias, obtida por MEV, do hidrogel constituído de PAAm e CMC (a) e dos bionanocompósitos com $10 \%$ (b) e $20 \%$ (c) de nanoargila com ampliações de 500 X.

A análise térmica foi utilizada para observar a temperatura inicial de degradação dos hidrogéis, com a finalidade de uma possível aplicação em solo. As curvas termogravimétricas (TG) e termogravimétricas diferenciais (DTG) dos hidrogéis analisados são apresentadas na Figura 3. Pode-se concluir que a nanoargila não influenciou significativamente a estabilidade térmica do material e que todas as amostras analisadas começam a degradar em aproximadamente $200^{\circ} \mathrm{C}$. Portanto, almejando uma possível aplicação como retentor de água ou fertilizante, ou em liberação controlada dos mesmos em solo, tais nanocompósitos apresentam excelentes estabilidades térmicas.

As curvas de DTG mostram cinco eventos de perda de massa, o primeiro (temperatura abaixo de $\left.200{ }^{\circ} \mathrm{C}\right)$ sendo atribuída a perda de água e voláteis, no segundo $\left(205,5-228,6{ }^{\circ} \mathrm{C}\right)$ ocorre a degradação dos grupamentos amidas da poliacrilamida e MBAAm, o terceiro (228,6 - 294,3 ${ }^{\circ} \mathrm{C}$ ) referindo-se à liberação de $\mathrm{H}_{2} \mathrm{O}, \mathrm{NH}_{3}$ e $\mathrm{CO}_{2}$ a partir da imidização, o quarto evento $\left(294,3\right.$ - 453,3 $\left.{ }^{\circ} \mathrm{C}\right)$ está relacionado com a ciclização das cadeias de poliacrilamida e/ou MBAAm e decomposição dos grupos funcionais $\left(\mathrm{CONH}_{2}\right.$ e $\left.\mathrm{COO}^{-}\right)$e por fim, o quinto evento $\left(453,3\right.$ - 604,2 $\left.{ }^{\circ} \mathrm{C}\right)$ está relacionado com a degradação completa do polímero (ZOLFAGHARI et al., 2006; LEITÃO et al., 2015; AOUADA et al., 2011; NATKANSKI et al., 2013; LIN et al., 2015). Para a nanoargila cloisita-Na+ é possível observar dois eventos térmicos principais. $\mathrm{O}$ primeiro evento ocorre entre $27,2-117,6^{\circ} \mathrm{C}$ com perda de massa de

Cultura Agronômica, Ilha Solteira, v.26, n.2, p.142-157, 2017 
aproximadamente $6,2 \%$. Este evento está relacionado à liberação de água e/ou outros materiais voláteis. $\mathrm{O}$ segundo evento, entre $544,7-715,2^{\circ} \mathrm{C}$, com perda de massa de $3,3 \%$, estando este relacionado à desidroxilação das camadas da cloisita- $\mathrm{Na}^{+}$. Esses resultados estão coerentes com os resultados apresentados por com Mallakpour e Dinari (2011).

Os valores das temperaturas iniciais, finais e máxima de decomposição, perda de massa de cada evento térmico e resíduo da nanoargila, hidrogel e seus nanocompósitos são mostrados na Tabela 1.

Tabela 1. Valores de temperaturas inicial $\left(\mathrm{T}_{\mathrm{i}}\right)$, final $\left(\mathrm{T}_{\mathrm{f}}\right)$ e máxima de perda de massa $\left(\mathrm{T}_{\mathrm{Max}}\right)$, e perda de massa e resíduo para cada evento das amostras estudadas.

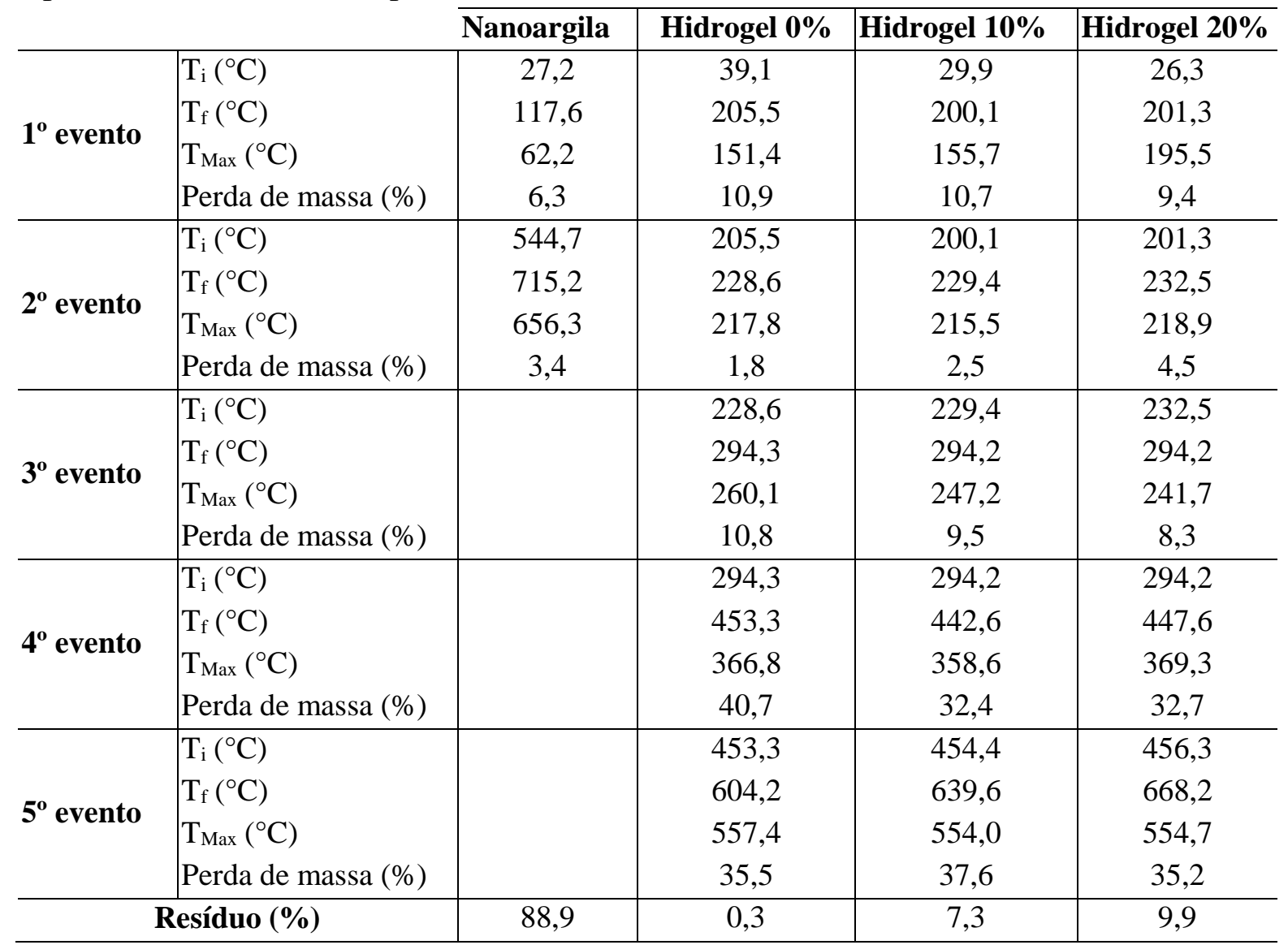

Cultura Agronômica, Ilha Solteira, v.26, n.2, p.142-157, 2017 

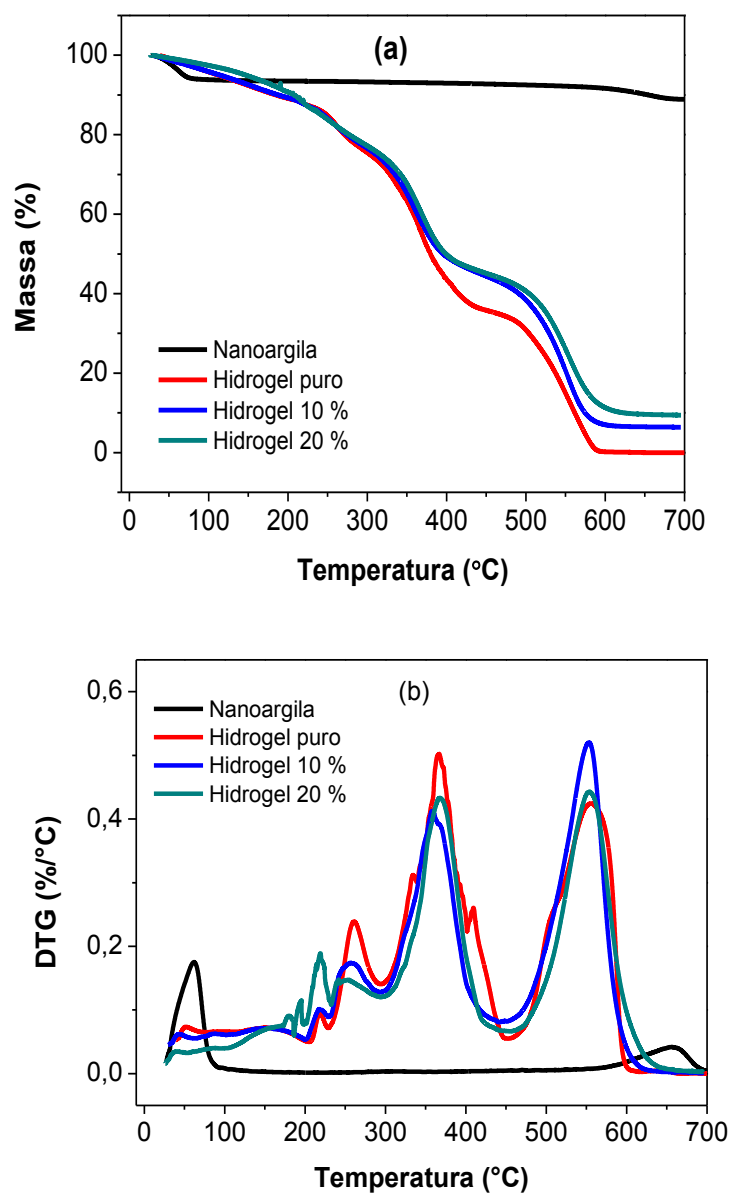

Figura 3. Curvas de TG (a) e DTG (b) da nanoargila, hidrogel puro e dos hidrogéis nanoestruturados com 10 e $20 \%$ de nanoargila.

A análise do grau de intumescimento (Q) é muito importante quando se almeja uma aplicação no ramo agronômico para determinado material, pois, com este parâmetro pode-se obter informações sobre a quantidade de água ou até mesmo soluções nutritivas que o bionanocompósito pode absorver. A absorção de água destilada foi medida por meio do grau de intumescimento (Q) em função do tempo, e o resultado de intumescimento em equilíbrio $\left(\mathrm{Q}_{\mathrm{eq}}\right)$ foi obtido através da não variação da massa do bionanocompósito em um determinado intervalo de tempo (Figura 4).

Cultura Agronômica, Ilha Solteira, v.26, n.2, p.142-157, 2017 

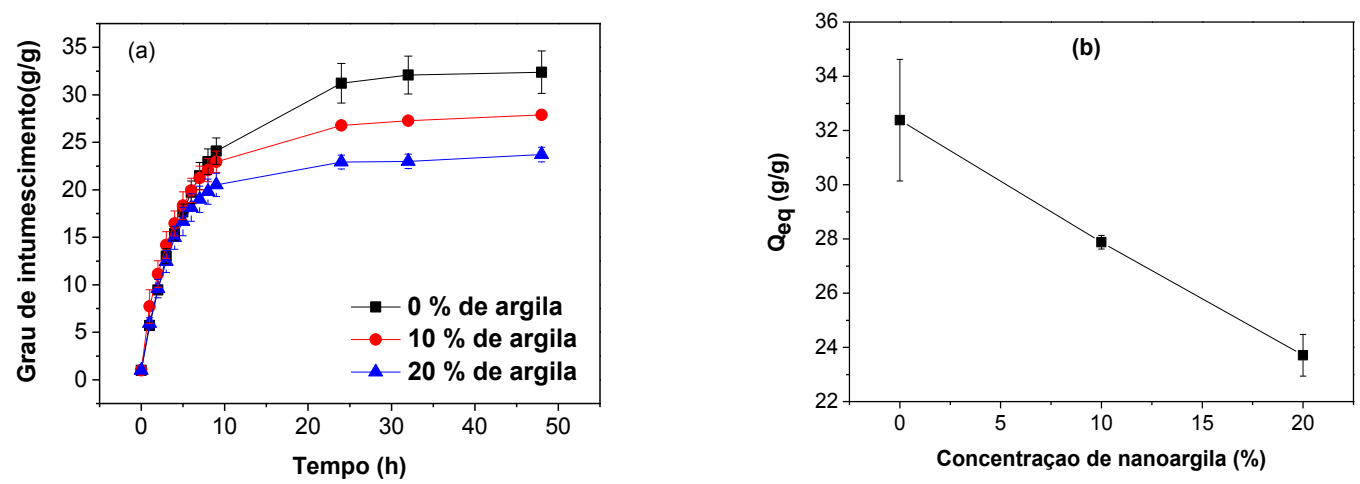

Figura 4. Grau de intumescimento (Q) em função do tempo para hidrogéis de AAm-CMC com diferentes concentrações de nanoargila (0-20\%) (a) e a dependência do grau de intumescimento no estado de equilíbrio em função da concentração de nanoargila (b).

Nota-se, que o aumento da concentração de nanoargila provoca diminuição do grau de intumescimento. É possível observar também uma rápida absorção de água durante as 9 primeiras horas de análises independente da concentração de argila nos bionanocompósitos. É importante ressaltar que após 48 horas de intumescimento os valores de $Q_{\text {eq }}$ se mantiveram constantes, pois a massa do bionanocompósito se manteve estável. Para o hidrogel composto com 0,10 e $20 \%$ de nanoargila, o valor de $Q_{\text {eq }}$ obtidos foram 32,38 $\pm 2,24 ; 27,88 \pm 0,25$ e $23,71 \pm 0,77 \mathrm{~g} / \mathrm{g}$, respectivamente. A diminuição do grau da capacidade de absorção de água dos bionanocompósitos com o incremento da concentração de nanoargila pode estar relacionado ao aumento do grau de reticulação nas cadeias do bionanocompósitos, o que contribui para o decréscimo da elasticidade das mesmas, e consequentemente, cadeias poliméricas menos elásticas alojam menores quantidades de água em seu interior (LEITÃO et al., 2015). Portanto, a nanocarga de argila atua provavelmente como reticulador físico e/ou químicos nas cadeias poliméricas formadoras dos hidrogéis (RASHIZADEH; OLAD, 2014). Tais informações são corroboradas com as informações das análises morfológicas.

O processo de intumescimento dos hidrogéis pode ser controlado pela variação de sua composição, pela densidade de reticulação e pelos fatores externos. Os resultados do grau de intumescimento em equilíbrio em função do tempo com relação à água destilada e diferentes concentrações salinas $(\mathrm{NaCl})$ para o hidrogel 0,10 e $20 \%$ de nanoargila (Figura 5a) mostraram uma diminuição no intumescimento em equilíbrio em soluções salinas de $\mathrm{NaCl}$ absorvendo aproximadamente 43 a $48 \%$ menos, quando comparado com o intumescimento em equilíbrio em água destilada. Tais resultados podem estar relacionados com uma diminuição na diferença da pressão osmótica entre o hidrogel e a solução externa (GUILHERME et al., 2015). Além disso, quando o hidrogel é imerso em soluções de $\mathrm{NaCl}$, os cátions provenientes da dissociação de $\mathrm{NaCl}$ podem interagir com os grupamentos carboxílicos e hidroxílicos das cadeias da carboximetilcelulose podendo formar pares iônicos $\left(\mathrm{COO}^{-} \mathrm{Na}^{+}\right.$e $\left.\mathrm{O}^{-} \mathrm{Na}^{+}\right)$. Assim, possíveis efeitos de repulsão eletrostática causada pelos grupos da carboximetilcelulose são minimizados dificultando a expansão das cadeias do hidrogel

Cultura Agronômica, Ilha Solteira, v.26, n.2, p.142-157, 2017 
(YADOLLAHI et al., 2015; ISLAM et al., 2016). Além disso, tais grupamentos hidrofílicos deixam de interagir com moléculas de água, o que diminui o grau de intumescimento.
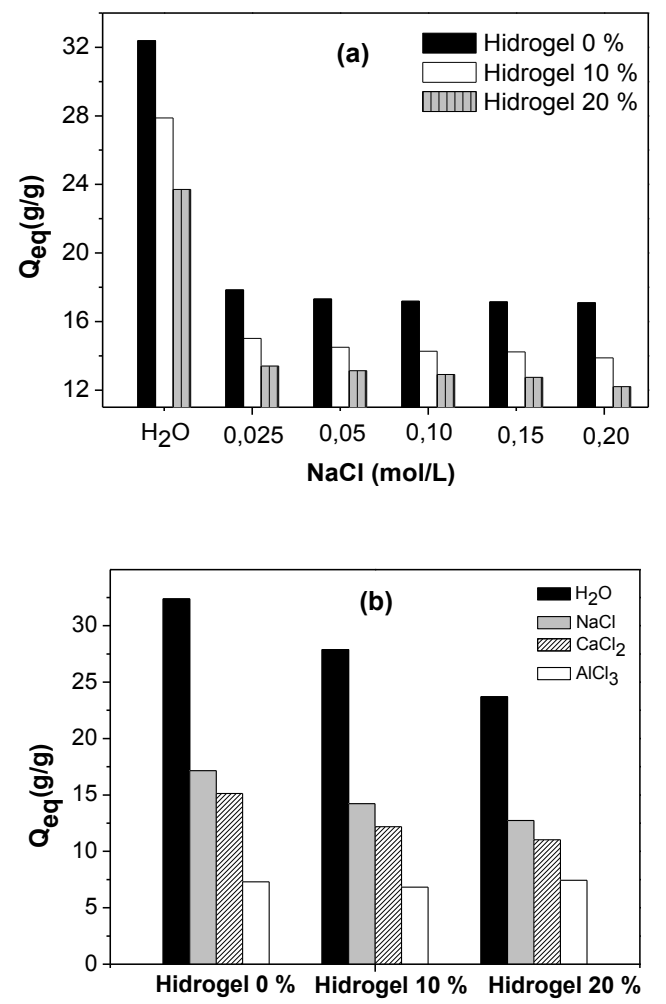

Figura 5. Dependência de $\mathrm{Q}_{\text {eq }}$ para hidrogel com 0, 10 e $20 \%$ de nanoargila em função do tempo para diferentes concentrações de $\mathrm{NaCl}$ (a) e cargas iônicas (b).

Foi estudado o efeito da carga iônica utilizando $0,15 \mathrm{~mol} / \mathrm{L}$ das soluções salinas de $\mathrm{NaCl}, \mathrm{CaCl}_{2}$ e $\mathrm{AlCl}_{3}$ no grau de intumescimento para as amostras contendo 0,10 e $20 \%$ de nanoargila (Figura 5b). Constatou-se um decréscimo nos valores de $\mathrm{Q}_{\mathrm{eq}}$ com o aumento da carga iônica para ambos as amostras analisadas. Tais resultados podem estar relacionados com a interação dos grupos carboxílicos e hidroxilas com os cátions $\left(\mathrm{X}^{\mathrm{n}+}\right)$ provenientes da dissociação das soluções salinas. Pois quanto maior a valência do contra-íon mais forte será as interações com os grupos aniônicos $(\mathrm{Y})$, ou seja: $\mathrm{YNa}<(\mathrm{Y})_{2} \mathrm{Ca}<(\mathrm{Y})_{3} \mathrm{Al}$. Além disso, os cátions trivalentes possuem uma alta densidade de cargas positivas podendo se ligar com três grupos carboxílicos, contribuindo ainda para a formação de pontos de reticulações física aumentando a rigidez das cadeias do hidrogel diminuindo assim, seu grau de intumescimento (BORTOLIN et al., 2012; LU et al., 2015; WAN et al., 2016). Vale ressaltar que as quantidades de água absorvidas no equilíbrio para as soluções de $\mathrm{NaCl}, \mathrm{CaCl}_{2}, \mathrm{e} \mathrm{AlCl}_{3}$ foram aproximadamente 46, 53 e $73 \%$ menores, quando comparado com a quantidade de água absorvida no grau de intumescimento no equilíbrio em água destilada. De maneira geral, redes de hidrogéis mais fortemente reticuladas e com alta densidade de reticulação absorvem menos água devido à sua pequena flexibilidade. Portanto, de acordo com os resultados, o grau de 
intumescimento foi fortemente influenciado pelas concentrações de solução salina e cargas iônicas quando comparado com o intumescimento em água destilada.

Os estudos dos parâmetros cinéticos foram avaliados com relação ao processo de difusão de água e soluções salinas para dentro da matriz do hidrogel. Para hidrogéis com formatos cilíndricos, são descritos três tipos de mecanismo de difusão: Fickiano: onde os valores de $\boldsymbol{n}$ estão entre 0,45 e 0,50 e quando os valores de $\boldsymbol{n}$ estão próximos de 1 é considerado um mecanismo de relaxamento de cadeias, anômalo: onde o mecanismo de difusão ocorre ao mesmo tempo por difusão e relaxação, e os valores de $\boldsymbol{n}$ encontram-se entre 0,50 e 0,89 (RITGER; PEPPAS, 1987; LU et al., 2015).

Os valores dos parâmetros cinéticos foram calculados através da equação 1, e a partir destes parâmetros, foram analisados o coeficiente de difusão $(\boldsymbol{D})$. No entanto, a equação 1 só pode ser aplicada desde o estado inicial até antes de atingir o equilíbrio da curva de $\ln \left(\mathrm{M}_{\mathrm{t}} / \mathrm{M}_{\mathrm{eq}}\right)$ vs $\ln$ t. Assim, os valores de $\boldsymbol{n}$ e $\boldsymbol{k}$ foram obtidos através dos coeficientes angulares e lineares, respectivamente (RITGER; PEPPAS, 1987; AOUADA et al., 2009).

Na Tabela 2 são listados os valores de $\boldsymbol{n}, \boldsymbol{k}$ e $\boldsymbol{D}$ obtidos a partir da curva de cinética de intumescimento dos hidrogéis e hidrogéis nanoestruturados intumescidos em água destilada e em diferentes soluções salinas. Com relação aos parâmetros cinéticos em água destilada, pode-se observar que o aumento da concentração de nanoargila provoca diminuição de $\boldsymbol{n}$ em relação ao hidrogel controle. Porém, todos os valores de $\boldsymbol{n}$ se mantiveram em 0,6 , indicando um processo de difusão mais Fickiano. Para os valores da constante de difusão $\boldsymbol{k}$ foi observado que a presença de nanoargila na matriz polimérica aumentou o mesmo, ou seja, a velocidade de absorção de água. Sendo, portanto um indício que o oxigênio ligado ao silício nas plaquetas de nanoargila pode estar interagindo com os grupamentos da cadeia do CMC e com a água destilada. Deste modo, a nanoargila atua também no mecanismo de absorção de água, acelerando tal processo. No entanto, intenta-se que mesmo acelerando a velocidade de absorção de água, a nanoargila atua como agente de reticulação contribuindo para a diminuição da quantidade de água total absorvida pela matriz, indicados em $\mathrm{Q}_{\mathrm{eq}}$.

Tabela 2. Parâmetros obtidos da cinética de intumescimento em água destilada e em diferentes soluções salinas $\left(\mathrm{NaCl}, \mathrm{CaCl}_{2}\right.$ e $\left.\mathrm{AlCl}_{3}\right)$.

\begin{tabular}{|c|c|c|c|c|c|c|c|c|c|}
\hline \multirow[b]{2}{*}{ Concentração } & \multicolumn{3}{|c|}{ Hidrogel $0 \%$} & \multicolumn{3}{|c|}{ Hidrogel $10 \%$} & \multicolumn{3}{|c|}{ Hidrogel $20 \%$} \\
\hline & $n$ & $\begin{array}{c}k \\
\left(h^{-1}\right)\end{array}$ & $\begin{array}{c}D \\
\left(10^{-3} \mathrm{~cm}^{2} / \mathrm{h}\right)\end{array}$ & $n$ & $\begin{array}{c}k \\
\left(h^{-1}\right)\end{array}$ & $\begin{array}{c}D \\
\left(10^{-3} \mathrm{~cm}^{2} / \mathrm{h}\right)\end{array}$ & $n$ & $\begin{array}{c}k \\
\left(h^{-1}\right) \\
\end{array}$ & $\begin{array}{c}D \\
\left(10^{-3} \mathrm{~cm}^{2} / \mathrm{h}\right)\end{array}$ \\
\hline $\mathrm{H}_{2} \mathrm{O}$ & 0,660 & 0,190 & 6,090 & 0,534 & 0,270 & 5,500 & 0,570 & 0,270 & 8,580 \\
\hline \begin{tabular}{l|l}
$\mathrm{NaCl}$ & $0,15 \mathrm{~mol} / \mathrm{L}$
\end{tabular} & 0,526 & 0,227 & 2,404 & 0,499 & 0,296 & 3,624 & 0,456 & 0,330 & 3,401 \\
\hline \begin{tabular}{l|l|}
$\mathrm{CaCl}_{2}$ & $0,15 \mathrm{~mol} / \mathrm{L}$
\end{tabular} & 0,527 & 0,233 & 2,671 & 0,473 & 0,311 & 2,885 & 0,421 & 0,399 & 3,476 \\
\hline \begin{tabular}{l|l}
$\mathrm{AlCl}_{3}$ & $0,15 \mathrm{~mol} / \mathrm{L}$
\end{tabular} & 0,391 & 0,423 & 1,802 & 0,344 & 0,473 & 1,504 & 0,322 & 0,457 & 1,116 \\
\hline
\end{tabular}

Nota-se também que os parâmetros cinéticos analisados são significativamente influenciados pela carga iônica da solução de intumescimento. Para as amostras de hidrogéis analisadas, observa-se que os valores de $\boldsymbol{n}$ diminuem com o aumento da carga iônica. Para os valores de $\boldsymbol{k}$, verificou-se que a velocidade de absorção aumenta significativamente com o 
aumento da carga iônica. Isto pode estar relacionado com a alta densidade de cargas positivas, acarretando em ligações fortes com a rede do hidrogel, proporcionando pontos de reticulações e aumentando a rigidez das cadeias (LIANG et al., 2009). Além disso, observa-se que o incremento do teor de argila provoca efeito semelhante nos parâmetros $\boldsymbol{n}$ e $\boldsymbol{k}$, o que corrobora com a hipótese de que pontos de reticulações afetam significativamente o mecanismo e a absorção de água pelos bionanocompósitos. E ainda, se analisarmos o $\boldsymbol{D}$, nota-se que os valores do coeficiente de difusão tende a diminuir com o aumento da carga iônica para ambos os hidrogéis analisados.

A constante de perda de água e a perda de água no equilíbrio foram analisadas com relação ao aumento da concentração de nanoargila (Tabela 3 ).

Tabela 3. Parâmetros obtidos da cinética de liberação de água com diferentes concentrações de nanoargila.

\begin{tabular}{c|c|c}
\hline Nanoargila & Perda de água (P.A) $(\boldsymbol{\%})$ & $\boldsymbol{k}\left(\mathbf{h}^{-\mathbf{1}}\right)$ \\
\hline $0 \%$ & $97,29 \pm 0,05$ & $35,06 \pm 1,43$ \\
\hline $10 \%$ & $96,91 \pm 0,14$ & $31,32 \pm 1,69$ \\
\hline $20 \%$ & $96,51 \pm 0,07$ & $27,32 \pm 1,25$ \\
\hline
\end{tabular}

Pode-se observar que o incremento de nanoargila na solução de síntese de hidrogel faz com que a perda de água (P.A.) e a velocidade de liberação de água (k) apresentam um decaimento, ou seja, os bionanocompósitos proporcionam uma liberação de água mais controlada em relação aos hidrogéis sem nanoargila. Portanto os hidrogéis analisados possuem boas propriedades de liberação de água, sendo um material promissor para uma possível utilização como controle de umidade de solos ou sistemas de liberação controlada de água no solo.

\section{CONCLUSÃO}

Os hidrogéis biodegradáveis e nanoestruturados foram obtidos com sucesso em diferentes teores de nanoargila. A difração de raios-x permite avaliar a conformação das plaquetas de nanoargila evidenciando a formação dos bionanocompósitos intercalados.

As micrografias eletrônicas mostram que as amostras são porosas, com tamanho de poros heterogêneos. Além disso, a presença da nanoargila na matriz polimérica produz um aumento da densidade de poros. A análise térmica apresenta uma degradação em aproximadamente $200{ }^{\circ} \mathrm{C}$ para todas as amostras estudadas.

As propriedades de intumescimento e cinéticas indicam uma grande dependência de nanocarga de argila e de carga iônica na matriz polimérica, devido ao aumento de reticulações acarretando em maior rigidez da cadeia, e a presença de nanoargila proporciona uma perda de água mais controlada. Assim os resultados evidenciam que os hidrogéis biodegradáveis e nanoestruturados são um produto promissor para a aplicação no ramo agrícola.

Cultura Agronômica, Ilha Solteira, v.26, n.2, p.142-157, 2017 


\section{AGRADECIMENTOS}

À Embrapa, Unesp e aos órgãos de fomento CAPES, CNPq e Fapesp pelo apoio financeiro e concessão de bolsas de produtividade em pesquisa e de estudo.

\section{REFERÊNCIAS BIBLIOGRÁFICAS}

AHMED, E. M. Hydrogel: Preparation, characterization and applications: A review. Journal of Advanced Research, Amsterdam, v. 6, n. 2, p.105-121, 2015.

AOUADA, F. A; MOURA, M. R; ORTS, W. J; MATTOSO, L. H. C. Preparation and characterization of novel micro - and nanocomposite hydrogels containing cellulosic fibrils. Journal of Agricultural and Food Chemistry, Washington, v. 59, n. 17, p.9433-9442, 2011. AOUADA, F. A.; MUNIZ, E. C.; MATTOSO, L. H. C. Correlação entre parâmetros da cinética de intumescimento com características estruturais e hidrofílicas de hidrogéis de poliacrilamida e metilcelulose. Química Nova, São Paulo, v. 32, n. 6, p.1482-1490, 2009.

BARDAJEE, G. R.; HOOSHYAR, Z. One-pot synthesis of biocompatible superparamagnetic iron oxide nanoparticles/hydrogel based on salep: Characterization and drug delivery. Carbohydrate Polymers, Oxford, v. 101, p.741-751, 2014.

BORTOLIN, A.; AOUADA, F. A.; LONG, E.; MATTOSO, L. H. C. Investigação do processo de absorção de água de hidrogéis de polissacarídeos: efeitos da carga iônica, presença de sais, concentrações de monômero e polissacarídeo. Polímeros, São Carlos, v. 22, n. 4, p.311-317, 2012.

CHAUHAN, S. Acrylic acid and methacrylic acid based hydrogels-A review. Der Chemica Sinica, Udaipur, v. 6, n. 1, p.61-72, 2015.

GUILHERME, M. R.; AOUADA, F. A.; FAJARDO, A. R.; MARTINS, A. F.; PAULINO, A. T.; DAVI, M. F. T.; RUBIRA, A. F.; MUNIZ, E. C. Superabsorbent hydrogels based on polysaccharides for application in agriculture as soil conditioner and nutrient carrier: A review. European Polymer Journal, Oxford, v. 72, p.365-385, 2015.

GONÇALVES, W. P.; SILVA, J; GOMES, J; MENEZES, R. R; NEVES, G. A; FERREIRA, H. C; SANTANA, L. N. L. Avaliação da influência de diferentes tratamentos térmicos sobre as transformações de fases esmectitas. Cerâmica, São Paulo, v. 60, n. 355, p.316-322, 2014.

ISLAM, T. M. D.; DAFADER, N. C.; PODDAR, P.; KHAN, N. M. D. S.; CHOWDHURY, A. M. S. Studies on swelling and absorption properties of the $\gamma$-irradiated polyvinyl alcohol (PVA)/kappa-carrageenan blend hydrogels. Journal of Advanced Chemical Engineering, Honnelles, v. 6, n. 2, p.1-6, 2016.

LEITÃO, R. C. F.; MOURA, C. P.; LINDOMAR da SILVA, R. D.; RICARDO, N. M. P. S.; FEITOSA, J. P. A.; MUNIZ, E. C.; FAJARDO, A. R.; RODRIGUES, F. H. A. Novel superabsorbent hydrogel composite based on poly(crylamide-co-acrylate)/nontronite: 
Characterization and swelling performance. Química Nova, São Paulo, v. 38, n. 3, p.370$377,2015$.

LI, P.; KIM, N. H.; SIDDARAMAJAH; LEE, J. H. Swelling behavior of polyacrilamide/laponite clay nanocomposite hydrogels: $\mathrm{pH}$-sensitive property. Composites Part. B: Engineering, Oxford, v. 40, n. 4, p.275-283, 2009.

LIANG, R.; YUAN, H.; XI, G.; ZHOU, Q. Synthesis of wheat straw-g-poly(acrylic acid) superabsorbent composites and release of urea from it. Carbohydrate Polymers, Oxford, v. 77, n. 2, p.181-187, 2009.

LIN, R; LI, A; LU, L; CAO, Y. Preparation of bulk sodium carboxymethyl cellulose aerogels with tunable morphology. Carbohydrate Polymers, Oxford, v. 118, p.126-132, 2015.

LU, J.; LI, Y.; HU, D.; CHEN, X.; LIU, Y.; WANG, L.; ZHAO, Y. Synthesis and properties of $\mathrm{pH}-$, thermo-, and salt-sensitive modified poly(asparticacid)/poly(vinylalcohol) IPN hydrogel and its drung controlled release. BioMed Research International, New York, v. 15, p.1-12, 2015. (Article ID 236745)

MALESU, V. K.; SAHOO, D.; NAYAK, P. L. Chitosan-sodium alginate nanocomposites blended with closite $30 \mathrm{~B}$ as a novel drug delivery system for anticancer drug curcumin. International Journal of Applied Biology and Pharmaceutical Technology, Hyderabad, v. 2, n. 3, p.402-411, 2011.

MALLAKPOUR, S.; DINARI, M. Biomodification of cloisite $\mathrm{Na}^{+}$with L-methionine amino acid and preparation of poly(vinyl alcohol)/organoclay nanocomposite films. Journal of Applied Polymer Science, Hoboken, v. 124, n. 5, p.4322-4330, 2012.

MARQUES, P. A. A.; CRIPA, M. A. M.; MARTINEZ, E. H. Hidrogel como substituto da irrigação complementar em viveiro telado de mudas de cafeeiro. Ciência Rural, Santa Maria, v. 43, n. 1, p.1-7, 2013.

MAZIAD, N. A.; EL FADL, F. I. A.; EL-KELESH, N. A.; EL-HAMOULY, S. H.; ZEID, I. F.; GAYED, H. M. Radiatin synthesis and characterization of super absorbent hydrogels for controlled release of some agrochemicals. Journal of Radioanalylitical and Nuclear Chemistry, Budapest, v. 307, n. 1, p.513-521, 2016

NASCIMENTO, D. W. S.; MOURA, M. R.; MATTOSSO, L. H. C.; AOUADA, F. A. Hybrid biodegradable hydrogels obtained from nanoclay and carboxymethylcellulose polysaccharide: hydrophilic, kinetic, spectroscopic and morphological properties. Journal of Nanoscience and Nanotechnology, Valencia, v. 17, n. 1, p.821-827, 2017.

NATKANSKI, P.; KÚSTROWSKI, P.; BIALAS, A.; PIWOWARSKA, Z.; MICHALIK, M. Thermal stability of montmorillonite polyacrylamide and polyacrylate nanocomposites and adsorption of Fe (III) ions. Applied Clay Science, Amsterdam, v. 76, p.153-157, 2013.

Cultura Agronômica, Ilha Solteira, v.26, n.2, p.142-157, 2017 
RASHIDZADEH, A.; OLAD, A. Slow-released NPK fertilizer encapsulated by NaAlg-gpoly(AA-co-AAm)/MMT superabsorbent nanocomposite. Carbohydrate Polymers, Oxford, v. 114, p.269-278, 2014.

RITGER, P. L.; PEPPAS, N. A. A simple equation for description of solute release II. Fickian and anomalous release from swellable devices. Journal of Controlled Release, Amsterdam, v. 5, n. 1, p.37-42, 1987.

SAVI, T.; MARIN, M.; BOLDRIN, D.; INCERTI, G.; ANDRI, S.; NARDINI, A. Green roofs for a drier world: Effects of hydrogel amendment on substrate and plant water status. Science of The Total Environment, Amsterdam, v. 490, p.467-476, 2014.

SAPUTRA, A. H.; HAPSSARI, M.; PITALOKA, A. B.; WULAN, P. P. D. K. Synthesis and characterization of hydrogel from cellulose derivatives of water hyacinth (Eichhormia crassipes) through chemical cross-linking method by using citric acid. Journal of Engineering Science and Technology, Selangor, v. 21, 75-86, 2015.

VUNDAVALLI, R.; VUNDAVALLI, S.; NAKKA, M.; RAO, D. S. Biodegradable nanohydrogels in agricultural farming-alternative source for water resources. Procedia Materials Science, Amsterdam, v. 10, 548-554, 2015.

WAN, T.; XIONG, J.; ZHAO, Q.; WU, D.; TANG, L.; LIAO, L.; CHEN, Q. Crosslinker effects on swelling and gel properties of $\mathrm{pH}$-and temperature-responsive poly (NIPAM/IA/AM) hydrogels. Polymer Bulletin, Hoboken, v. 73, n. 5, p.1447-1458, 2016.

YADOLLAHI, M.; GHOLAMALI, I.; NAMAZI, H.; AGHAZADEH, M. Synthesis and characterization of antibacteral carboxymethylcellulose/CuObio-nanocomposite hydrogels. International Journal of Biological Macromolecules, Amsterdam, v. 73, p. 109-114, 2015.

ZOLFAGHARI, R.; KATBAB, A. A.; NABAVIZADEH, J.; YOUSEFZADEH, R.; NEJAD, M. H. Preparation and characterization of nanocomposite hydrogels based on polyacrylamide for enhanced oil recovery applications. Journal of Applied Polymer Science, Hoboken, v. 100, n. 3, p.2096-2103, 2006. 